\title{
Qualitative Evaluation of the Bracket-Adhesive-Enamel Junction of Metal Orthodontic Brackets: A Preliminary Report
}

\author{
${ }^{1}$ Department of Pediatric Dentistry, School of \\ Dentistry, University of Detroit Mercy, USA, Former \\ Professor Department of Pediatric Dentistry and \\ Orthodontics, College of Dentistry, King Saud \\ University, Riyadh, Saudi Arabia, ${ }^{2}$ Saudi Board \\ Orthodontic Resident, King Saud University, Saudi \\ Arabia, ${ }^{3}$ Orthodontic Resident, Boston University \\ Henry M. Goldman School of Dental Medicine, \\ Massachusetts, US, ${ }^{4}$ Department of Pediatric \\ Dentistry and Orthodontics, College of Dentistry, \\ King Saud University, Riyadh, Saudi Arabia \\ Correspondence: \\ salamafs@udmercy.edu \\ Tel.: + 3134946877 \\ Fax: + 3134946781
}

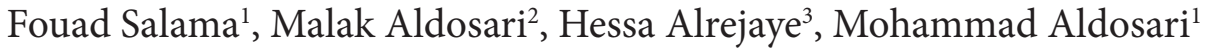

Received: 29 December 2018; Accepted: 30 July 2019 bracket-adhesive-enamel junction. sives, all groups showed partial or complete overlap of the adhesive on the bracket. No statistical difference was found between the groups for adhesive overlap $(\mathrm{P}=1.0)$. However, resin-modified glass-ionomer was found to be statistically significantly $(\mathrm{P}<0.05)$ better than composite resin in both smoothness and the lack of projections or depressions, regardless of the instrument of removal. Conclusion. Removing excess adhesive with a dental explorer or a micro brush is not an ideal method for adhesive removal, as partial or complete overlap of the adhesive on the bracket existed in all groups. On the other hand, resin-modified glassionomer was a superior material to composite resin for better smoothness and surface topography at the

Key Words: Orthodontic Brackets - Surface Topography - Surface Characteristics - Orthodontic Adhesive.

\section{Introduction}

Some of the features of ideal orthodontic adhesives are: sufficient working time for the dentist, fluoride release, good bonding, and easy removal without damaging the enamel surface, with minimal polishing needed (1$3)$. Another ideal feature that has not been tested before is the easy removal of the adhesive from the areas around the enamel and orthodontic bracket, after bonding and before setting, anticipating a smooth surface. Composite resin is the most commonly used adhesive because of its well-established clinical and laboratory performance $(4,5)$. On the other hand, resin-modified glass-iono- 
mer cements have some advantages, which include its ability to form a chemical bond with the enamel and metal, less sensitivity to moisture and saliva contamination, fluoride release, and the ability to serve as a fluoride reservoir $(6,7)$.

White spot lesions due to decalcification of the enamel surface adjacent to fixed orthodontic appliances are a frequent undesirable consequence of orthodontic treatment $(8,9)$. Several studies have reported the prevalence of white spot lesions to be as high as 96 percent (10-13). Fixed appliances and bonding materials increase the retention of biofilm and the development of white spot lesions $(8,9)$. Management of white spot lesions on the enamel surface comprises a variety of approaches to prevent demineralization, and procedures to promote remineralization of any demineralization present $(8,14)$. A wide variety of methods has been described and implemented for the prevention of such lesions, implemented by the patient and/or the dentist $(8,9,14)$. However, not all methods to prevent white spot lesions are successful (15).

The application of adhesives to enamel and orthodontic brackets could be a source of irregular and rough surfaces at the bracket-adhesive-enamel junction after adhesive removal, and could create areas for plaque accumulation, with the resultant demineralization $(11,16,17)$. The surface roughness of various metal and esthetic brackets, as well as various orthodontic wires, has been tested. However, to our knowledge no published research has evaluated the surface topography and roughness of the areas around the enamel and the orthodontic bracket after bonding and adhesive removal.

Therefore, the aim of this study was to assess qualitatively the surface topography at the bracket-adhesive-enamel junction using a digital microscope, after bonding to the buccal and lingual surfaces of premolars, using two orthodontic adhesives: composite resin (Transbond XT, 3M Unitek, Monrovia, California, USA) and resin-modified glassionomer (Fuji Ortho LC, GC Corporation, Tokyo, Japan), and two adhesive removal methods, using a dental explorer and a micro brush. The null hypothesis tested in this study was that there is no difference in the surface topography at the bracket-adhesiveenamel junction after removal of the two orthodontic adhesives using a dental explorer or a micro brush.

\section{Materials and Methods}

\section{Preparation of Specimens}

The study protocol was approved by the Research and Ethical Committee of Human Studies at the College of Dentistry Research Center. Twenty human premolar teeth extracted due to orthodontic treatment, stored in $0.1 \%$ thymol solution, were used in this investigation. The teeth included had an intact crown, no attrition, and were free from hypoplastic areas, cracks, gross irregularities, decay and fractures. The enamel surface of each tooth was scaled and polished with a rubber polishing cup and pumice using a low- speed handpiece for 10 seconds, then stored in deionized water at room temperature $\left(27^{\circ} \mathrm{C}\right)$ for 48 hours. The apical part of each root was mounted in self-curing acrylic resin (Vertex $^{\text {Tx }}$ Orthoplast, Vertex-Dental B. V. Asia Pte Ltd, Singapore) to facilitate perpendicular sectioning of each tooth into two sections (buccal and lingual), and then each section was decoronated $4 \mathrm{~mm}$ below the CEJ using a diamond saw mounted under a water spray (IsoMet-2000 Precision Saw, Buehler, Lake Bluff, IL, USA). Each section/ surface was then placed in a standardized mold and embedded in self-curing acrylic resin, where the buccal and lingual surfaces of each tooth were kept parallel to the floor. The teeth were allocated into four groups, with 10 specimens/group, according to the 
adhesive material and removal methods used, with 20 buccal and 20 lingual surfaces (Table 1). Due to the difficulty of collecting teeth and the limited number, the lingual and buccal surfaces of each tooth were used to increase the sample size. The power sample size was 0.81 and the level of significant $\sigma=0.05$, with estimated standard deviation $=0.9$. The sample size should be at least 9 in each group.

\section{Bracket Bonding and Evaluation}

Orthodontic premolar brackets with gingival offset (Ortho Classic, Roth 0.022, Ortho Classic Inc., McMinnville, OR, USA) were positioned using firm and even pressure, and bonded to the enamel surface, following the manufacturer's recommendations, using 2 types of orthodontic adhesive: composite resin (3M-Unitek Transbond ${ }^{\mathrm{m}}$ XT Light Cure Adhesive, Monrovia, CA, USA) for groups 1 and 2, and resin- modified glassionomer (GC Fuji Ortho LC Capsule, GC Corporation, Tokyo, Japan) for groups 3 and 4. The brackets were then bonded onto the mesio-distal and occluso-cervical center of the tooth surfaces. Excess adhesive was removed in 5 applications using a regular size micro applicator brush \#2 (Dental Micro Applicator Brush, Shanghai Smedent Medical Instrument Co., Ltd., Shanghai, China) or a dental explorer (Double Ended \#5, HuFriedy Mfg. Co., LLC, Chicago, USA). One micro brush was used for each specimen and was wiped with gauze after each application. In addition, the explorer was also wiped with gauze after each application. The specimens were light cured using an Ortholux ${ }^{\mathrm{xw}}$ Luminous Curing Light (3M Unitek Orthodontic Products, Monrovia, CA, USA) (App. $1600 \mathrm{~mW} / \mathrm{cm}$ ) (LED). One investigator performed all the bonding procedures in a consistent manner and random order. The specimens were stored in distilled water at room temperature for 48 hours. Each speci- men was then photographed using a digital microscope (Digital Microscope System Model KH 7700, Hirox; USA, Inc., Hackensack, NJ, USA) at fifty times magnification. One photograph, which included the bracket and the surrounding enamel, was taken. Before the evaluation started, the two expert evaluators discussed the evaluation scores and they were calibrated. The two independent examiners did not know the group under evaluation. Each photograph was evaluated and the bracket-adhesive-enamel junction scored at the occlusal edge/side of the bracket. The following parameters were assessed: the adhesive overlap of the bracket, the smoothness of the enamel, and the presence of projections and depressions. The adhesive overlap of the bracket was allocated to one of three classifications: no overlap, where no adhesive was covering any part of the occlusal edge/side of the bracket; partial overlap, where there was adhesive covering any part less than the entire length of the occlusal edge/side of the bracket; and complete overlap, where the adhesive covered the entire length of the occlusal edge/side of the bracket. The smoothness of the enamel was given one of three possible classifications: completely smooth enamel - when no rough area of adhesive was present over the entire length of the occlusal edge/side of the bracket; partially smooth enamel - when some rough areas of adhesive were present but less than the entire length of the occlusal edge/side of the bracket; and no smooth enamel - when a rough area of adhesive was present along the entire length of the occlusal edge/side of the bracket. The presence of projections and depressions on the adhesive was given one of four possible classifications: no projections and depressions, projections present, depressions present, and both projections and depressions present. For all parameters, when disagreement was recorded between the two examiners, the highest score was recorded. 


\section{Statistical Analysis}

Comparison of different groups and identifying statistically significant differences were performed using the Fisher's exact test. The statistical significance was set at a $p$ value of $<0.05$. Inter-examiner reliability for scoring different parameters at the bracketadhesive-enamel junction was completed using the Kappa statistic. All procedures were performed using Stata SE15.1 software (StataCorp, College Station, TX, USA).

\section{Results}

Inter-examiner reliability for scoring different parameters showed that the Kappa statistic was 0.74 , which indicated high agreement. Regardless of the adhesive removal method used, all groups showed partial or complete overlap of the adhesive on the bracket (Table 1).

Groups in which a composite resin was used on the buccal surfaces showed complete overlap of the adhesive in $90 \%$ of the surfaces when the dental explorer was used, and $100 \%$ of the surfaces when the micro brush was used (Table 1). Groups in which a resin-modified glass-ionomer was used on the lingual surfaces showed complete overlap of the adhesive on the bracket in $100 \%$ of the surfaces when an explorer was used, and $90 \%$ of the surfaces when a micro brush was used to remove the excess material (Table 1). The Fisher-exact statistical test showed no significant difference between the groups for complete/partial overlap of the adhesive on the bracket on the buccal or lingual surfaces $(\mathrm{P}=1.0)$.

A completely rough surface of adhesive on the enamel was found for the composite resin when the dental explorer (10\%) or micro brush (30\%) were used, while none was found for the resin-modified glass-ionomer (Table 1). A partially rough surface of adhesive on the enamel was found for the composite resin when the dental explorer (90\%) or micro brush (70\%) were used, while for the resin-modified glass-ionomer $50 \%$ of rough surface was recorded when the dental

Table 1. Frequency of the Three Outcomes between the Removal Methods and Adhesive Materials

\begin{tabular}{|c|c|c|c|c|c|c|}
\hline \multirow{3}{*}{ Outcomes } & \multicolumn{6}{|l|}{ Groups } \\
\hline & \multicolumn{3}{|c|}{ Dental Explorer $(n=20)$} & \multicolumn{3}{|c|}{ Micro brush $(n=20)$} \\
\hline & $\begin{array}{l}\mathrm{CR} \\
(\mathrm{N}=10)\end{array}$ & $\begin{array}{l}\text { RMGI } \\
(\mathrm{N}=10)\end{array}$ & $\begin{array}{l}P \\
\text { value }\end{array}$ & $\begin{array}{l}\mathrm{CR} \\
(\mathrm{N}=10)\end{array}$ & $\begin{array}{l}\text { RMGI } \\
(\mathrm{N}=10)\end{array}$ & $\begin{array}{l}P \\
\text { value }\end{array}$ \\
\hline \multicolumn{7}{|c|}{ Overlap of the adhesive over the bracket $(\mathrm{N}=40)$} \\
\hline No overlap & 0 & 0 & \multirow{3}{*}{1.0} & 0 & 0 & \multirow{3}{*}{1.0} \\
\hline Partial overlap & 1 & 0 & & 0 & 1 & \\
\hline Complete overlap & 9 & 10 & & 10 & 9 & \\
\hline \multicolumn{7}{|c|}{ Smoothness of the adhesive on the enamel $(\mathrm{N}=40)$} \\
\hline Completely smooth & 0 & 5 & \multirow{3}{*}{$0.033^{*}$} & 0 & 5 & \multirow{3}{*}{$0.016^{*}$} \\
\hline Partially smooth & 9 & 5 & & 7 & 5 & \\
\hline Completely rough & 1 & 0 & & 3 & 0 & \\
\hline \multicolumn{7}{|c|}{ Presence of projections and depressions on the adhesives $(\mathrm{N}=40)$} \\
\hline None present & 0 & 9 & \multirow{4}{*}{$<0.001^{*}$} & 0 & 6 & \multirow{4}{*}{$0.001^{*}$} \\
\hline Projections & 3 & 0 & & 1 & 0 & \\
\hline Depressions & 0 & 1 & & 0 & 2 & \\
\hline Both present & 7 & 0 & & 9 & 2 & \\
\hline
\end{tabular}

$\mathrm{CR}=$ Composite Resin; RMGl=Resin-Modified Glass lonomer; P-value of 2-sided Fisher's exact test; *Significant at $\mathrm{P}<0.05$. 
explorer or and $40 \%$ when micro brush were used (Table 1). Resin-modified glass-ionomer was statistically significantly better for a completely smooth adhesive surface on the enamel compared to resin composite, when using the dental explorer $(50 \% ; \mathrm{P}=0.033)$ or the micro brush (50\%; $\mathrm{P}=0.016)$.

Composite resin adhesive displayed both projections and depressions in $70 \%$ of the specimens when dental explorer was used, and $90 \%$ when a micro brush was used to clean the adhesive residues. On the other hand, resin-modified glass-ionomer presented superior topography, where only $10 \%$ of specimens had depressions when the explorer was used $(\mathrm{P}<0.001)$, and $40 \%$ $(\mathrm{P}=0.001)$ had some form of surface topography when using a micro brush (Table 1 ).

\section{Discussion}

The null hypothesis tested in this study was rejected, as there was a difference in the surface topography at the bracket-adhesiveenamel junction after the removal of two orthodontic adhesives, using a dental explorer or a micro brush. In the present study, regardless of the method used to remove the adhesives on the buccal or lingual surfaces, no group prevented overlap of the adhesive on the bracket and all groups showed partial or complete overlap of the adhesive. The measures reported for prevention of white spot lesions have rarely considered the use of orthodontic adhesives and their possible overlap on the brackets as a potential source of formation of irregular and rough surfaces at the bracket-adhesive-enamel junction after adhesive removal (14). The forces of adhesion of cariogenic bacteria to the enamel, the adhesive and the stainless steel bracket were found to be lowest on the enamel and highest on the adhesive $(18,19)$. Morphological changes in the topography of the enamel, especially roughness, are of considerable clinical importance for the formation of white spot lesions (20). One study reported that, even with relatively uniform surface roughness, surface free energy was significantly different between resin composite and resin-modified glass-ionomer adhesives, which affects the adhesion of Streptococcus mutans (21). Resin-modified glass-ionomers showed significantly higher surface free energy than composite resin (21).

In the present study, there was a significant difference between the groups in the distribution of the smoothness of the adhesive on the enamel surface, and a rough surface was found on the composite resin when a dental explorer or a micro brush was used, while none was found on the resinmodified glass-ionomer. In contrast, more completely smooth adhesive was found using the resin-modified glass-ionomer when a dental explorer or a micro brush was used, and none was found for the composite resin. Again, these results indicate that the application of adhesives for bonding orthodontic brackets to the enamel could be a source of irregular and rough surfaces at the bracketadhesive-enamel junction after adhesive removal. This may restrict cleansing activity and increase the risk of white spot formation (14). This scenario is supported by a study which reported the lower occurrence of white spot caries lesions in patients who used orthodontic lingual appliances, due to their self-cleansing activity (22).

A rough surface provides opportunities for bacterial adhesion by increasing the surface area and providing suitable niches (23, 24). Differences in surface roughness were reported, with the greater surface roughness of different materials used for brackets than orthodontic adhesives, which was attributed to minor dissimilarities in the surface roughness $(<0.5 \mu \mathrm{m})$ of different materials (24). However, another study showed that minor variations in surface roughness had no significant effect on the contact angles for surface free energy, or on the adhe- 
sion of bacteria $(23,25)$. The adherence of Streptococcus mutans to a fixed appliance is largely the result of the bracket material, where titanium brackets had the least number of Streptococcus mutans and the greatest number of Candida albicans (26). The variations in the adhesion of Streptococcus mutans between brackets have been attributed to the surface free energy and tension (26). It has been shown that stainless steel had more surface tension, which is demonstrated by the more likely attachment of microorganisms on metallic brackets compared with ceramic brackets $(26,27)$. Accordingly, substrates/materials with high surface free energy will attract more microorganisms to their surfaces than those with less surface free energy (26). In the present study, there was a significant difference between the groups in terms of the presence of projections and depressions on the adhesive, and both projections and depressions were present in the adhesive in composite resin specimens when a dental explorer or a micro brush was used, while fewer projections and depressions were found in the resin-modified glass-ionomer when a micro brush was used, and no projections and depressions were found when a dental explorer was used. The differences in the distribution of diverse surface irregularities shown in this study may be due to the dissimilar characteristics and composition of each adhesive and orthodontic material surface. This is supported by the studies reporting the influence of surface roughness on the adhesion of bacteria to the surfaces $(11,16,17)$. In addition, it has been suggested that the type of filler modifies the surface of orthodontic composite resin (28). However, another study reported that the filler size, volume and composition of different composite resins have no effect on the adhesion of microorganisms (29). It has been reported that glass ionomer and resin composites have a tendency to collect more plaque or microor- ganisms than different restorative materials in vivo and in vitro (30). The adhesion of microorganisms to the orthodontic composite resin is attributed to van der Waals forces, as well as hydrophobic and electrostatic factors, and Streptococcus mutans adhesion to composite resin is greater than to orthodontic appliances (31). In the present study, excess adhesive was removed from around the brackets during the bonding process to simulate clinical practice. Another study compared the effects on bonding strength of removing excess adhesive from around the bracket base both immediately after placing the bracket on the tooth and after subjecting the adhesive to 5 seconds of light curing to initially secure the bracket in its proper position, and concluded that removing excess adhesive after 5 seconds of light cure significantly decreased the bond strength at 24 hours (19). It has been reported that the adhesive properties influence the consistency of the bond, the ease of debonding and the ease of cleaning the enamel (32).

The design of this study had multiple dimensions, which included the effects of the tooth surface, adhesive type, and the adhesive removal method. Due to the difficulty of collecting teeth, we used the lingual and buccal surfaces of each tooth to increase the sample size. We also used the buccal surfaces for composite resin, and the lingual surfaces for resin-modified glass-ionomer orthodontic adhesives. This is supported by a study, which reported no significant differences in shear bond strength between the buccal and lingual surfaces of premolars, and resin-modified glass-ionomer and composite resin adhesives exhibited sufficient shear bond strength for orthodontic use, with no significant difference between the two adhesives (33).

\section{Limitations of the Study}

The present study has some limitations, such as the use of only two orthodontic 
adhesives. It would be beneficial to compare more orthodontic adhesives. We also only evaluated the occlusal edge/side of the bracket, which may be easier to clean compared to other edges/sides. In addition, aging of specimens by thermocycling, to imitate the conditions of the oral cavity, was not performed. It would be useful to expose the specimens to thermocycling and saliva to simulate the oral environment. In addition, use of a brush versus a micro brush, and finishing of the adhesives, were not tested in this study. Further research is needed for evaluation of the different methods used to improve the smoothness of the surface at the bracket-adhesive-enamel junction so that it is less attractive to biofilm. In addition, future investigations are necessary to analyze different parameters of the surfaces, such as surface roughness, and the composition of each material on microbial adhesion.

\section{Conclusion}

Within the limits of this in vitro study, it was concluded that removing excess adhesive with a dental explorer or a micro brush is not an ideal method for adhesive removal, as partial or complete overlap of the adhesive on the bracket existed in all groups. Resin-modified glass-ionomer was a superior material over composite resin for better smoothness and surface topography at the bracket-adhesive-enamel junction. There was a difference in the surface topography at the bracket-adhesive-enamel junction between the methods used in this study. There was a significant difference between the groups in terms of distribution of the smoothness of the adhesives, as well as the presence of projections and depressions on the adhesives.

What Is Already Known on this Topic:

The application of adhesives to enamel and orthodontic brackets could be a source of irregular and rough surfaces at the bracket-adhesive-enamel junction after adhesive removal, and could create areas for plaque accumulation, with the resultant demineralization. The surface roughness of various metal and esthetic brackets, as well as different orthodontic wires, have been tested. However, to our knowledge no published research has evaluated the surface topography and roughness of the areas around the enamel and the orthodontic bracket after bonding and adhesive removal.

\section{What this Study Adds:}

The manuscript provides evidence-based research to support what orthodontists practice every day. As no published research has previously evaluated the surface topography of the areas around the enamel and the orthodontic bracket after bonding, and adhesive removal using different adhesive removal methods, this study aimed to assess qualitatively the surface topography at the bracket-adhesive-enamel junction after bonding using two orthodontic adhesives and the use of two adhesive removal methods.

Acknowledgement: We would like to thank the College of Dentistry Research Center and the Deanship of Scientific Research at King Saud University, Saudi Arabia, for supporting this research. The authors wish to express sincere thanks to Mr. Nassr Al Maflehi for his valuable help in the statistical analysis.

Authors' Contributions: Conception and design: FS; Acquisition, analysis and interpretation of data: FS, MA, and HA; Drafting the article: FS, MA, HA, and MA; Revising it critically for important intellectual content: FS, MA, HA, and MA; Approved final version of the manuscript: FS, MA, HA, and MA.

Conflict of Interest: The authors declare that they have no conflict of interest.

\section{References}

1. Pithon MM, Oliveira MV, Ruellas AC, Bolognese AM, Romano FL. Shear bond strength of orthodontic brackets to enamel under different surface treatment conditions. J Appl Oral Sci. 2007;15(2):127-30

2. Shinya M, Shinya A, Lassila LV, Gomi H, Varrela J, Vallittu PK, et al. Treated enamel surface patterns associated with five orthodontic adhesive systems--surface morphology and shear bond strength. Dent Mater J. 2008;27(1):1-6.

3. Chicri RO, Sasaki RT, Carvalho AS, Nouer PR, Lima-Arsati YB. Effect of enamel pretreatment on shear bond strength of brackets bonded with resin-modified glass-ionomer cement. World J Orthod. 2010;11(1):11-5. 
4. Romano FL, Correr AB, Sobrinho LC, Borges de Araújo Magnani MB, Vieira de Siqueira VC. Shear bond strength of metallic brackets bonded with a new orthodontic composite. Braz J Oral Sci. 2009;8(2):76-80.

5. Finnema KJ, Ozcan M, Post WJ, Ren Y, Dijkstra PU. In-vitro orthodontic bond strength testing: a systematic review and meta-analysis. Am J Orthod Dentofacial Orthop. 2010;137(5):615-22.e3.

6. Coonar AK, Jones SP, Pearson GJ. An ex vivo investigation into the fluoride release and absorption profiles of three orthodontic adhesives. Eur J Orthod. 2001;23(4):417-24.

7. Hamed MM, Tawfek ZS, Younis MT. Shear bond strength of resin modified glass ionomer cement using different enamel conditions. Al-Rafidain Dent J. 2010;10(1):127-32.

8. Sudjalim TR, Woods MG, Manton DJ. Prevention of white spot lesions in orthodontic practice: a contemporary review. Aust Dent J. 2006;51(4):284-9; quiz 347.

9. Heymann GC, Grauer D. A contemporary review of white spot lesions in orthodontics. J Esthet Restor Dent. 2013;25(2):85-95.

10. Mizrahi E. Enamel demineralization following orthodontic treatment. Am J Orthod. 1982;82(1):62-7.

11. Gorelick L, Geiger AM, Gwinnett AJ. Incidence of white spot formation after bonding and banding. Am J Orthod. 1982;81(2):93-8.

12. Ogaard B, Rølla G, Arends J, ten Cate JM. Orthodontic appliances and enamel demineralization. Part 2. Prevention and treatment of lesions. Am J Orthod Dentofacial Orthop. 1988;94(2):123-8.

13. Mitchell L. Decalcification during orthodontic treatment with fixed appliances--an overview. $\mathrm{Br}$ J Orthod. 1992;19(3):199-205.

14. Morrier JJ. White spot lesions and orthodontic treatment. Prevention and treatment [in French]. Orthod Fr. 2014;85(3):235-44.

15. Chen H, Liu X, Dai J, Jiang Z, Guo T, Ding Y. Effect of remineralizing agents on white spot lesions after orthodontic treatment: a systematic review. Am J Orthod Dentofacial Orthop. 2013;143(3):376-82. e3.

16. Ogaard B. Prevalence of white spot lesions in 19-year-olds: a study on untreated and orthodontically treated persons 5 years after treatment. Am J Orthod Dentofacial Orthop. 1989;96(5):423-7.

17. Rosenbloom RG, Tinanoff N. Salivary Streptococcus mutans levels in patients before, during, and after orthodontic treatment. Am J Orthod Dentofacial Orthop. 1991;100(1):35-7.
18. Mei L, Busscher HJ, van der Mei HC, Chen Y, de Vries J, Ren Y. Oral bacterial adhesion forces to biomaterial surfaces constituting the bracket-adhesive-enamel junction in orthodontic treatment. Eur J Oral Sci. 2009;117(4):419-26.

19. Bishara SE, VonWald L, Olsen ME, Laffoon JF. Comparisons of two approaches for removing excess adhesive during the bonding procedure. Angle Orthod. 2000;70(2):149-53.

20. Ferreira FG, Nouer DF, Silva NP, Garbui IU, Correr-Sobrinho L, Nouer PR. Qualitative and quantitative evaluation of human dental enamel after bracket debonding: a noncontact three-dimensional optical profilometry analysis. Clin Oral Investig. 2014;18(7):1853-64.

21. Ahn SJ, Lim BS, Lee SJ. Surface characteristics of orthodontic adhesives and effects on streptococcal adhesion. Am J Orthod Dentofacial Orthop. 2010;137(4):489-95; discussion 13A.

22. van der Veen MH, Attin R, Schwestka-Polly $\mathrm{R}$, Wiechmann D. Caries outcomes after orthodontic treatment with fixed appliances: do lingual brackets make a difference? Eur J Oral Sci. 2010;118(3):298-303.

23. Brusca MI, Chara O, Sterin-Borda L, Rosa AC. Influence of different orthodontic brackets on adherence of microorganisms in vitro. Angle Orthod. 2007;77(2):331-6.

24. Lee SP, Lee SJ, Lim BS, Ahn SJ. Surface characteristics of orthodontic materials and their effects on adhesion of mutans streptococci. Angle Orthod. 2009;79(2):353-60.

25. Bollen CM, Papaioanno W, Van Eldere J, Schepers E, Quirynen M, van Steenberghe D. The influence of abutment surface roughness on plaque accumulation and peri-implant mucositis. Clin Oral Implants Res. 1996;7(3):201-11.

26. Rammohan SN, Juvvadi SR, Gandikota CS, Challa P, Manne R, Mathur A. Adherence of Streptococcus mutans and Candida albicans to different bracket materials. J Pharm Bioallied Sci. 2012;4(Suppl 2):S212-6.

27. Eliades T, Eliades G, Brantley WA. Microbial attachment on orthodontic appliances: I. Wettability and early pellicle formation on bracket materials. Am J Orthod Dentofacial Orthop. 1995;108(4):351-60.

28. Ahn SJ, Lim BS, Yang HC, Chang YI. Quantitative analysis of the adhesion of cariogenic streptococci to orthodontic metal brackets. Angle Orthod. 2005;75(4):666-71.

29. Saku S, Kotake H, Scougall-Vilchis RJ, Ohashi S, Hotta M, Horiuchi S, et al. Antibacterial activity 
of composite resin with glass-ionomer filler particles. Dent Mater J. 2010;29(2):193-8.

30. Derks A, Katsaros C, Frencken JE, van't Hof MA, Kuijpers-Jagtman AM. Caries-inhibiting effect of preventive measures during orthodontic treatment with fixed appliances. A systematic review. Caries Res. 2004;38(5):413-20.

31. Velazquez-Enriquez U, Scougall-Vilchis RJ, Contreras-Bulnes R, Flores-Estrada J, Uematsu S, Yamaguchi R. Adhesion of Streptococci to vari- ous orthodontic composite resins. Aust Dent J. 2013;58(1):101-5.

32. Clark SA, Gordon PH, McCabe JF. An ex vivo investigation to compare orthodontic bonding using a 4-META-based adhesive or a composite adhesive to acid-etched and sandblasted enamel. J Orthod. 2003;30(1):51-8; discussion 23.

33. Salama F, Aldosari M, Alrejae H, Almosa N. Shear bond strength of orthodontic bracket bonded to buccal versus lingual surfaces. IOSR J Dent Med Sci (IOSR-JDMS). 2018;17(1):24-9. 